\title{
Comparison of Miller laryngoscope and UEScope videolaryngoscope for endotracheal intubation in four pediatric airway scenarios: a randomized, crossover simulation trial
}

\author{
Jacek Smereka ${ }^{1} \cdot$ Marcin Madziala $^{2} \cdot$ Dominika Dunder $^{2} \cdot$ Elzbieta Makomaska-Szaroszyk $^{2} \cdot$ Lukasz Szarpak $^{2}$ (D)
}

Received: 13 July 2018 / Revised: 21 March 2019 / Accepted: 26 March 2019 / Published online: 11 April 2019

(C) The Author(s) 2019

\begin{abstract}
With different videolaryngoscopes for pediatric patients available, UEScope can be used in all age groups. The aim of this study was to compare the Miller laryngoscope and UEScope in pediatric intubation by paramedics in different scenarios. Overall, 93 paramedics with no experience in pediatric intubation or videolaryngoscopy performed endotracheal intubation in scenarios: (A) normal airway without chest compressions, (B) difficult airway without chest compressions, (C) normal airway with uninterrupted chest compressions, (D) difficult airway with uninterrupted chest compressions. Scenario A. Total intubation success with both laryngoscopes: $100 \%$. First-attempt success: $100 \%$ for UEScope, $96.8 \%$ for Miller. Median intubation time for UEScope: $13 \mathrm{~s}$ [IQR, 12.5-17], statistically significantly lower than for Miller: $14 \mathrm{~s}$ [IQR, 12-19.5] ( $p=0.044)$. Scenario B. Total efficacy: $81.7 \%$ for Miller, $100 \%$ for UEScope $(p=0.012)$. First-attempt success: $48.4 \%$ for Miller, $87.1 \%$ for UEScope $(p=0.001)$. Median intubation time: $27 \mathrm{~s}$ [IQR, 21-33] with Miller, $15 \mathrm{~s}$ [IQR, 14-21] with UEScope $(p=0.001)$. Scenario C. Total efficiency: $91.4 \%$ with Miller, $100 \%$ with UEScope $(p=0.018)$; first-attempt success: 67.7 vs. $90.3 \%(p=0.003)$, respectively. Intubation time: $21 \mathrm{~s}$ [IQR, 18-28] for Miller, $15 \mathrm{~s}$ [IQR, 12-19.5] for UEScope. Scenario D. Total efficiency: 65.6\% with Miller, 98.9\% with UEScope $(p<0.001)$; first-attempt success: 29.1 vs. $72 \%(p=0.001)$, respectively. Intubation time: $38 \mathrm{~s}$ [IQR, $23-$ 46] for Miller, $21 \mathrm{~s}$ [IQR, 17-25.5] for UEScope.

Conclusion: In pediatric normal airway without chest compressions, UEScope is comparable with Miller. In difficult pediatric airways without chest compressions, UEScope offers better first-attempt success, shorted median intubation time, and improved glottic visualization. With uninterrupted chest compressions in normal or difficult airway, UEScope provides a higher firstattempt success, a shorter median intubation time, and a better glottic visualization than Miller laryngoscope.

\section{What is Known:}

- Endotracheal intubation is the gold standard for adult and children airway management.

- More than two direct laryngoscopy attempts in children with difficult airways are associated with a high failure rate and increased incidence of severe complications.

What is New:

- In difficult pediatric airways with or without chest compressions, UEScope in inexperienced providers in simulated settings provides better first-attempt efficiency, median intubation time, and glottic visualization.
\end{abstract}

Keywords Cardiopulmonary resuscitation $\cdot$ Endotracheal intubation $\cdot$ Pediatric $\cdot$ Physician $\cdot$ Videolaryngoscopy

Communicated by Piet Leroy

Lukasz Szarpak

lukasz.szarpak@gmail.com

Jacek Smereka

smereka.icu@gmail.com

Marcin Madziala

mmadziala262@gmail.com
Dominika Dunder

src.emergency@gmail.com

Elzbieta Makomaska-Szaroszyk

ptmk.kontakt@gmail.com

Extended author information available on the last page of the article 


\section{Abbreviations}

CONSORT Consolidated Standards of Reporting Trials POGO percentage of glottic opening

\section{Introduction}

In pediatric acute care settings, endotracheal intubation is the golden standard for securing the airway in situations where the provider is unable to ventilate the patient adequately with a bag-and-mask or by a supraglottic airway device, or if an open airway is compromised. Cases of inability to perform bagmask ventilation or to obtain correct placement of supraglottic airway devices, especially among non-anesthesiological personnel or in patients with difficult airway, emphasize the role of videolaryngoscopes [3]. The pediatric and adult airway anatomy and physiology differ, and medical personnel experience in direct pediatric intubation can be limited [23].

A complication of failed endotracheal intubation is hypoxemia [4]. A way to reduce the potential complications, including airway edema, is to decrease the number of total intubation attempts and the procedure duration, which is possible with better or alternative intubation technique, including videolaryngoscopy $[14,18]$. Fiadjoe et al. suggest that more than two direct laryngoscopy attempts in children with difficult airway are associated with a high rate of failure and severe complications [4]. Moreover, direct pediatric laryngoscopy is bound with significant interruptions in chest compressions [2].

Videolaryngoscopy offers a better viewing angle and less traumatic intubation, can reduce the complication rate $[15,17$, 30], and supports intubation training [13].

Many different videolaryngoscopes for pediatric patients are available, but not all can be used in all age groups [23, 30]. UEScope is a portable videolaryngoscope [29] invented in 2010, equipped with several blades for different age groups. Its specific feature is the angulated blade [29]. The technique for introducing UEScope does not demand tongue sweep. UEScope has been tested in various clinical conditions and most of the studies were performed in Chinese pediatric patients $[23,24]$. The results, though inconsistent, suggest that UEScope is not inferior to direct laryngoscopy. As is true for many videolaryngoscopes, the UEScope learning curve is better than that for direct laryngoscopy. Owing to the slim design and low weight, UEScope is easy to manipulate [29].

Videolaryngoscopy with the use of numerous devices in difficult pediatric airway with continuous chest compressions has been tested in several studies. Comparing GlideScope and direct laryngoscopy in a pediatric simulator by novice physicians, Rabiner et al. found that GlideScope did not improve time to intubation or intubation success rates in normal or difficult airway scenarios [16]. In turn, in a study by Szarpak et al. concerning pediatric endotracheal intubation with chest compressions, the first-attempt intubation and overall intubation success were better with GlideScope than with Miller direct laryngoscopy [25]. TruView offered better intubation conditions than Macintosh in a pediatric manikin scenario with chest compressions with and without cervical stabilization [24].

The UEScope was selected because it is a portable device with angulated or Miller's blade which can be used in all age group and the results concerning effectiveness in endotracheal intubation obtained so far were inconsistent.

The aim of the study was to compare the Miller laryngoscope and UEScope during pediatric intubation performed by paramedics in different airway conditions.

\section{Methods}

The study was designed as a prospective, multicenter, randomized, crossover simulation trial. It was performed in Warsaw, Wroclaw, and Poznan, Poland, between December 2017 and May 2018, in accordance with the Consolidated Standards of Reporting Trials (CONSORT) [17]. The protocol was approved by the Institutional Review Board of the Polish Society of Disaster Medicine (approval No. 21.11.2017.IRB).

\section{Participants}

The study included 93 paramedics participating in Pediatric Advance Life Support courses based on the American Heart Association guidelines. Voluntary written informed consent was obtained from all participants. The inclusion criteria were the following: (1) not having performed more than 100 clinical adult intubations with direct laryngoscopy and no experience in clinical pediatric intubation; (2) no experience in clinical or experimental training in videolaryngoscopy [24]. Wrist or back injury up to 1 month before the study was the exclusion criterion.

\section{Scenarios}

To simulate endotracheal tube immediate airway management conditions in a 5-year-old child, the Pediatric HAL $\AA$ S3005 simulator was used (Gaumard® Scientific, Miami, USA). The participants performed intubation in four scenarios:

1. Scenario A: normal airway without chest compressions.

2. Scenario B: difficult airway without chest compressions. The simulator control software inflated the tongue to simulate conditions of Mallampati scale grade III [18].

3. Scenario C: normal airway with uninterrupted chest compressions. To standardize the difficulties resulting from chest compressions, the Corpuls system (GS Elektromedizinische Geräte G. Stemple GmbH, 
Kaufering, Germany) was used to apply chest compressions (depth, $5 \mathrm{~cm}$; frequency, 100 per minute).

4. Scenario D: difficult airway with uninterrupted chest compressions. As in scenario B, tongue inflation was applied. The Corpuls system performed chest compressions as in scenario $\mathrm{C}$.

In all scenarios, the simulator was placed on a flat surface in a well-lit room with the head in neutral position. In both tested devices, the blade size No. 2 was used. In both tested devices, Miller's blade was used. Endotracheal intubation was performed with a standard sealed tube of 5.5 internal diameter. A stylet was used, moistened with a lubricant, like the endotracheal tube itself. The shape of the stylet was standard and the tip of the stylet was bent to form a $90^{\circ}$ angle. After each intubation attempt, the participant had to confirm ventilation correctness using a self-inflating bag (Ambu, Copenhagen, Denmark).

\section{Study protocol}

Prior to the study, all paramedics participated in a 30-min training, covering the anatomy, physiology, and pathophysiology of the pediatric respiratory tract, as well as endotracheal intubation with the tested devices. The training with the Miller laryngoscope (Heine USA Ltd., Dover, USA) and UEScope (Zhejiang UE Medical Corp., Zhejiang, China) was performed by an experienced anesthesiologist (Fig. 1). Then, the participants had up to $10 \mathrm{~min}$ to practice intubation with the tested devices in normal adult airways with the AT Kelly Torso manikin (Laerdal, Stavanger, Norway).

One week after the training, the paramedics performed endotracheal intubation in the pediatric patient in various scenarios of simulated cardiopulmonary resuscitation using the tested devices.

The order of participants and intubation methods was random: the Research Randomizer program (randomizer.org) was used. Figure 2 presents the detailed randomization procedure. Each participant had up to three attempts to intubate with each laryngoscope. Then a 10-min break was taken before the participant performed the intubation scenarios with the remaining intubation device. The paramedics were informed that the patient required immediate endotracheal intubation.

\section{Measurements and outcomes}

The primary outcome was first intubation attempt success, defined as effective ventilation with an endotracheal tube, accompanied by a chest rise in the simulator and air flow in the lungs recorded by the simulator software. The following criteria determined failed intubation: more than three

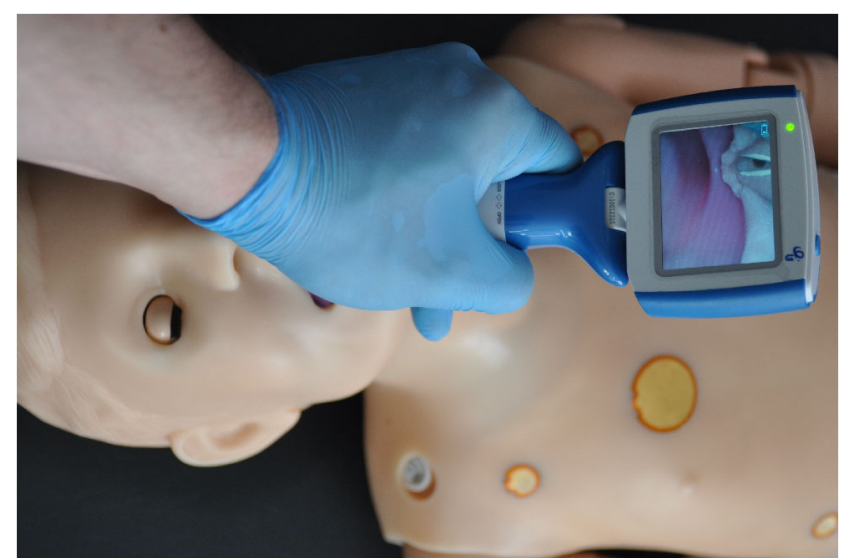

Fig. 1 Intubation with a UEScope videolaryngoscope

unsuccessful attempts, intubation procedure exceeding $120 \mathrm{~s}$, or unrecognized esophageal intubation.

The secondary outcomes included intubation time, time to best glottic view, percentage of glottic opening and the Cormack-Lehane grade, and the ease of intubation. The intubation time was defined as the time from grasping the laryngoscope to the first attempt of effective ventilation with a selfexpanding bag. The measured "time to best glottic view" was defined as "I can see the vocal cords." The study participants assessed the glottic visibility degree: using percentage of glottic opening (POGO) [28] and the Cormack-Lehane grade [5]. A $100 \%$ POGO score indicates visualization of the entire glottic, while a $0 \%$ POGO score means no visualization of the laryngeal structures. After each intubation, the paramedics also described the ease of intubation on a scale from 1 (very easy) to 100 (very difficult).

\section{Sample size calculation and statistical analysis}

The sample size was calculated with the G*Power 3.1 software, and the two-tailed $t$ test was applied (Cohen's $d, 0.8$; alpha error, 0.05; power, 0.95). The minimum of 61 participants turned out necessary to achieve an acceptable level of significance and power of the study; 93 were involved.

\section{Results}

\section{Demographics}

The study involved 93 paramedics (26 female; $27.9 \%$ ) with no experience in videolaryngoscopy. All were employees of Emergency Medical Service teams. Their median age was 32 years [IQR, 29-37.5], and median work experience in emergency medicine equaled 6.5 years [IQR, 4-9]. 
Fig. 2 Time to intubation during the study

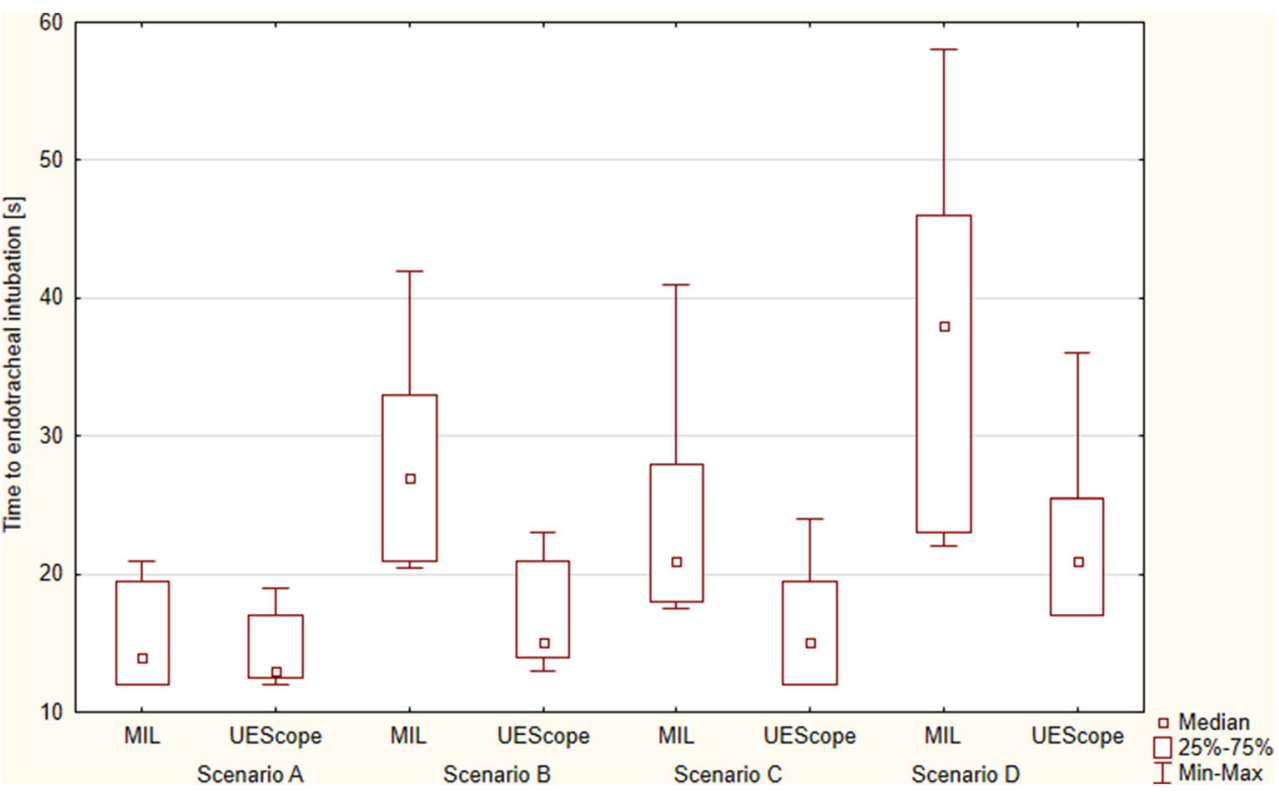

\section{Scenario A: normal airway without chest compressions}

The overall success with UEScope as well as with Miller was $100 \%$. The first-attempt success was $100 \%$ for UEScope, and $96.8 \%$ for Miller (Table 1). The median intubation time for UEScope was $13 \mathrm{~s}$ [IQR, 12.5-17], lower than with Miller (14 s [IQR, 12-19.5]) ( $p=0.044$; Fig. 2).

Intubation using UEScope compared with Miller was associated with a better glottic visualization based on the Cormack-Lehane scale $(p=0.012)$. The POGO score was 81\% [IQR, 75-93] for Miller and 87\% [IQR, 85-100] for UEScope ( $p=0.037$; Fig. 3$)$. Intubation with UEScope proved easier in the subjective assessment as compared with Miller (15 [IQR, 10-19] vs. 18 points [IQR, 15-22]) $(p=$ 0.021; Fig. 4).

\section{Scenario B: difficult airway without chest compressions}

The total endotracheal intubation efficacy with Miller and UEScope varied and equaled 81.7 vs. $100 \%$, respectively $(p=0.012)$. Successful endotracheal intubation on firstattempt success was $48.4 \%$ for Miller and $87.1 \%$ for UEScope ( $p=0.001$; Table 1). The median intubation time was $27 \mathrm{~s}$ [IQR, 21-33] for Miller and $15 \mathrm{~s}$ [IQR, 14-21] for UEScope ( $p=0.001$; Fig. 2 ). The degree of glottic visualization based on the Cormack-Lehane scale proved better for UEScope $(p<0.001)$. The POGO score was $25 \%$ [IQR, 2333 ] for Miller and $80 \%$ [IQR, 74-90] for UEScope $(p<0.001$; Fig. 3). The ease of use amounted to 74 points [IQR, 68-82] for Miller and 27 points [IQR, 16-25] for UEScope $(p<0.001$; Fig. 4).

\section{Scenario C: normal airway with uninterrupted chest compressions}

The overall success was $91.4 \%$ with Miller and $100 \%$ with UEScope $(p=0.018)$, with the successful endotracheal intubation on first-attempt success 67.7 vs. $90.3 \%$, respectively $(p=0.003$; Table 1). The intubation time was $21 \mathrm{~s}[\mathrm{IQR}, 18$ 28] for Miller and $15 \mathrm{~s}$ [IQR, 12-19.5] for UEScope. The UEScope procedure was statistically significantly shorter than that with Miller ( $p=0.005$; Fig. 2). Glottic visualization with UEScope compared with Miller was also statistically significantly better $(p=0.011)$. The POGO score equaled $61 \%$ [IQR, 50-72] for Miller and 81\% [IQR, 80-98] for UEScope $(p<0.001$; Fig. 3). The intubation ease with UEScope was 17 points [IQR, 14-23], statistically significantly better than that with Miller (39 points [IQR, 31-53]) $(p<0.001$; Fig. 4).

\section{Scenario D: difficult airway with uninterrupted chest compressions}

The intubation efficiency was higher for UEScope compared with Miller both overall $(98.9$ vs. $65.6 \% ; p<0.001)$ and for the first attempt (72 vs. $29.1 \% ; p=0.001)$. UEScope proved superior to Miller also with regard to the procedure duration (21 [IQR, 17-25.5] vs. 38 s [IQR, 23-46]; $p=0.004$; Fig. 2), glottic visualization based on the Cormack-Lehane scale $(p<0.001)$, POGO score (75\% [IQR, 68-89] vs. $21 \%$ [IQR, 14-27]; $p<0.001$; Fig. 3), and ease of use (30 [IQR, 28-39] vs. 85 points [IQR, 77-92]; $p<0.001$; Fig. 4). 
Table 1 Study outcomes by laryngoscope devices $(n=93)$

\begin{tabular}{l} 
Outcome \\
\hline Scenario A: normal airway without chest \\
Success of intubation attempts [\%] \\
1st \\
2 nd \\
3 rd \\
Overall intubation success rate [\%] \\
Time to endotracheal intubation [s] \\
Cormack and Lehane grade \\
1 \\
2 \\
3 \\
4 \\
POGO score, 1-100
\end{tabular}

Miller laryngoscope

UEScope videolaryngoscope

$p$ value

Ease of use, 1-100

$93(100 \%)$

$-$

$93(100 \%)$

$93(100 \%)$

13 (12.5-17)

$93(100 \%)$

$-$

$-$

$-$

87 (85-100)

15 (10-19)

0.037

0.021
Scenario B: difficult airway without chest compressions

Success of intubation attempts [\%]

1 st
2nd
3rd

Overall intubation success rate [\%]

$45(48.4 \%)$

$21(22.6 \%)$

$10(10.7 \%)$

Time to endotracheal intubation [s]

$76(81.7 \%)$

$27(21-33)$

Cormack and Lehane grade

1

2

3

4

POGO score, 1-100

Ease of use, 1-100

Scenario C: normal airway with chest compressions

Success of intubation attempts [\%]

$1 \mathrm{st}$
$2 \mathrm{nd}$
$3 \mathrm{rd}$

Overall intubation success rate [\%]

$63(67.7 \%)$

$20(21.5 \%)$

$2(2.2 \%)$

Time to endotracheal intubation [s]

$85(91.4 \%)$

$21(18-28)$

Cormack and Lehane grade

1

2

$43(46.2 \%)$

$39(41.9 \%)$

$10(10.7 \%)$

4

POGO score, 1-100

$61(50-72)$

39 (31-53)

Ease of use, 1-100

Scenario D: difficult airway with chest compressions

Success of intubation attempts [\%]

$\begin{array}{ll}\text { 1st } & 27(29.1 \%) \\ \text { 2nd } & 31(33.3 \%) \\ \text { 3rd } & 3(3.2 \%) \\ \text { Overall intubation success rate [\%] } & 61(65.6 \%) \\ \text { Time to endotracheal intubation [s] } & 38(23-46)\end{array}$

Cormack and Lehane grade

$\begin{array}{ll}81(87.1 \%) & 0.001 \\ 12(12.9 \%) & \\ - & \\ 93(100 \%) & 0.012 \\ 15(14-21) & 0.001 \\ & \\ 51(54.8 \%) & <0.001 \\ 39(42 \%) & \\ 3(3.2 \%) & \\ - & <0.001 \\ 80(74-90) & <0.001 \\ 27(16-35) & \end{array}$

$84(90.3 \%)$

$9(6.7 \%)$

$-$

$93(100 \%) \quad 0.018$

$15(12-19.5) \quad 0.005$

$90(96.8 \%) \quad 0.011$

$3(3.2 \%)$

$-$

$81(80-98) \quad<0.001$

$17(14-23) \quad<0.001$

$67(72 \%)$

$20(21.5 \%)$

$5(5.4 \%)$

$92(98.9 \%)$

$<0.001$

21 (17-25.5)

0.004 
Table 1 (continued)

\begin{tabular}{llll}
\hline Outcome & Miller laryngoscope & UEScope videolaryngoscope & $p$ value \\
\hline 1 & - & $47(50.5 \%)$ & $<0.001$ \\
2 & $7(7.5 \%)$ & $37(39.8 \%)$ & \\
3 & $70(75.3 \%)$ & $9(9.7 \%)$ & \\
4 & $16(17.2 \%)$ & - & $<0.001$ \\
POGO score, $1-100$ & $21(14-27)$ & $75(68-89)$ & $<0.001$ \\
Ease of use, $1-100$ & $85(77-92)$ & $30(28-39)$ & \\
\hline
\end{tabular}

$N S$ not statistically significant, $P O G O$ percentage of glottis opening

\section{Discussion}

The use of videolaryngoscopy in normal and difficult airway has been widely tested in adults. However, videolaryngoscopes are still not regarded as the standard first-attempt devices for normal airway intubation in children $[6,10,22]$. Their application depends on the clinical settings and the intubator's experience. Many studies have been carried out on the role of videolaryngoscopy in normal and difficult airway in pediatric patients $[12,18,23,24,30,32]$. Some suggest that videolaryngoscopes offer no benefit over direct laryngoscopy performed by emergency department personnel with regard to the rate of first-pass intubation success, complications, or successful intubation [3]. Other studies reveal advantages of videolaryngoscopy in children, especially with difficult airway $[9,16,20,24]$.

In our study in normal airway without chest compressions, the median intubation time was comparable, and the differences were statically significant although not relevant from a practical point of view. In difficult airway without chest compressions, the total endotracheal intubation efficacy and median intubation time using Miller and UEScope varied, and the differences were statistically and practically important. In normal airway with uninterrupted chest compressions, those differences were also statistically important. The biggest differences were found in difficult airway with uninterrupted chest; they were statistically significant and noticeable practical significance.

UEScope has been tested in different clinical conditions in adult and pediatric patients, generally Chinese; the role of UEScope for non-Chinese patients is raised [7, 8, 21, 22, 31, 32], with the consideration of potential differences in airway anatomy. These studies were published mainly in Chinese, and their results are available thanks to publications by Xue et al. [29, 30].

Most studies concerning videolaryngoscopes in pediatric patients analyzed normal airway [19]. We investigated various situations, including difficult airway and intubation accompanied by continuous chest compressions.

Although inexperienced providers are not expected to perform pediatric intubation in their daily practice routinely, comparing personnel more experienced in direct laryngoscopy than in the use of videolaryngoscopy would be a source of methodological bias.

Wan [24] proved a better glottic view, a higher first-attempt success rate, and shorter intubation time with UEScope than
Fig. 3 Percentage of glottic opening (POGO score)

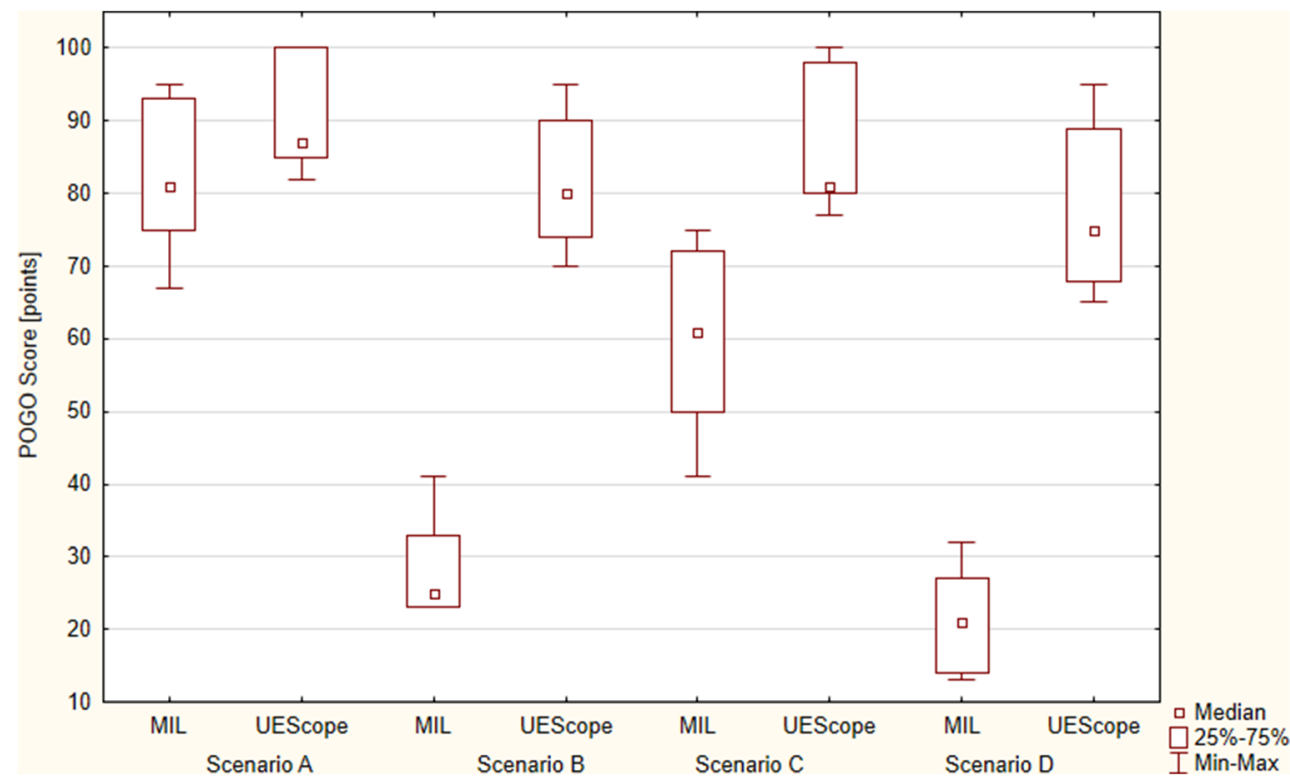


Fig. 4 Ease of intubation with different airway scenarios

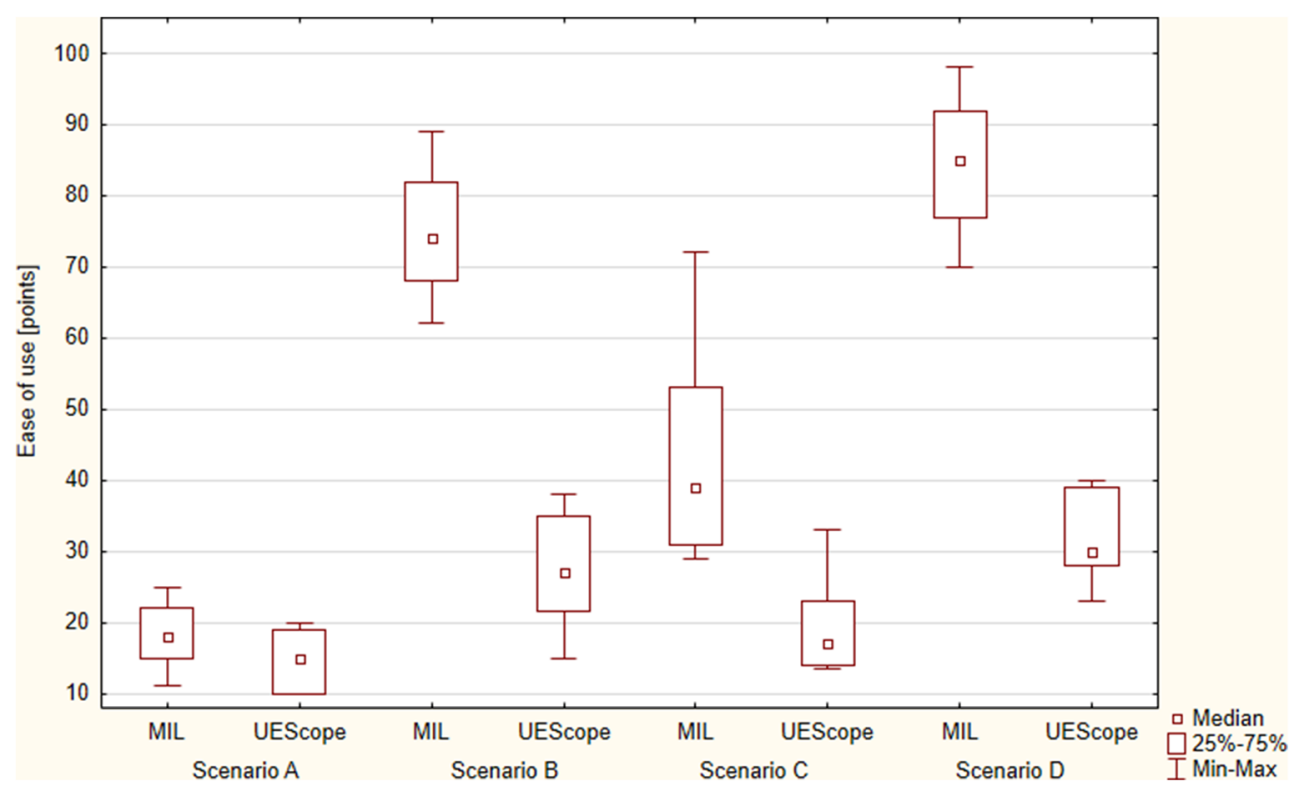

with Macintosh in normal airway pediatric patients. In our simulation, endotracheal tube placement was equal in UEScope and direct laryngoscopy groups, but still the UEScope intubation time turned out shorter than that with direct laryngoscopy. The differences can be explained by the assumed definitions of time to intubation and the clinical vs. simulation setting.

In a study by Jiang and Jin [8] among 200 children with normal airway, videolaryngoscopy improved the laryngeal view and the success rate compared with Macintosh, but the intubation time was similar. We found a shorter intubation time in the difficult airway scenario, comparable with that in the clinical study by Jiang and Jin.

UEScope was also compared with direct laryngoscopy for endotracheal intubation in neonates for planned surgery. Its first-attempt success was non-inferior or better and the intubation time was shorter [1,26, 27, 31]. The size of the groups in these studies was limited and the intubator's experience; this can be the reason for the first-attempt success inconsistency.

\section{Limitations and strengths}

One of the limitations is that the study was performed with a pediatric simulator. However, medical simulation allows for multiple procedures with no potential harm to the patient, and for standardization of conditions [11, 21]. Secondly, the study group was limited to paramedics. Although being inexperienced intubators, they work in emergency situations and face pediatric patients, including those requiring advanced airway management. The third limitation is the shorter learning curve and better glottic visualization with the use of videolaryngoscopy comparing to direct laryngoscopy.
The study strengths include the randomization, crossover design, comparison of various scenarios, and advanced research methods.

\section{Conclusions}

In pediatric normal airway without chest compressions, UEScope is comparable with Miller. In difficult pediatric airways without chest compressions, UEScope offers better firstattempt success, shorted median intubation time, and improved glottic visualization. With uninterrupted chest compressions in normal or difficult airway, UEScope provides a higher first-attempt success, a shorter median intubation time, and a better glottic visualization than Miller laryngoscope.

Acknowledgments We would like to thank all the participants for their involvement in our study.

Authors' contributions JS, MM, and LS contributed significantly to the planning of the study and the study design. All authors recruited the participants and collected the data. JS and LS were the principal investigators of this study and did major manuscript preparation. LS and JS prepared the statistical analysis. JS, MM, DD, EMS, and LS contributed significantly to manuscript editing and expertise.

\section{Compliance with ethical standards}

Conflict of interests The authors declare that they have no conflict of interest.

Ethical approval Approval was granted by the Institutional Review Board of the Polish Society of Disaster Medicine (approval no. 21.11.2017.IRB). 
Informed consent Informed consent was obtained from all participants included in the study.

Source of support No sources of financial or material support to be declared.

Open Access This article is distributed under the terms of the Creative Commons Attribution 4.0 International License (http:// creativecommons.org/licenses/by/4.0/), which permits unrestricted use, distribution, and reproduction in any medium, provided you give appropriate credit to the original author(s) and the source, provide a link to the Creative Commons license, and indicate if changes were made.

\section{References}

1. Donoghue AJ, Ades AM, Nishisaki A, Deutsch ES (2013) Videolaryngoscopy versus direct laryngoscopy in simulated pediatric intubation. Ann Emerg Med 61(3):271-277. https://doi.org/ 10.1016/j.annemergmed.2012.09.008

2. Donoghue A, Hsieh TC, Nishisaki A, Myers S (2016) Tracheal intubation during pediatric cardiopulmonary resuscitation: a videography-based assessment in an emergency department resuscitation room. Resuscitation. 99:38-43. https://doi.org/10.1016/j. resuscitation.2015.11.019

3. Eisenberg MA, Green-Hopkins I, Werner H, Nagler J (2016) Comparison between direct and video-assisted laryngoscopy for intubations in a pediatric emergency department. Acad Emerg Med 23(8):870-877. https://doi.org/10.1111/acem.13015

4. Fiadjoe JE, Nishisaki A, Jagannathan N, Hunyady AI, Greenberg RS, Reynolds PI, Matuszczak ME, Rehman MA, Polaner DM, Szmuk P, Nadkarni VM, McGowan FX Jr, Litman RS, Kovatsis PG (2016) Airway management complications in children with difficult tracheal intubation from the Pediatric Difficult Intubation (PeDI) registry: a prospective cohort analysis. Lancet Respir Med 4(1):37-48. https://doi.org/10.1016/S2213-2600(15)00508-1

5. Glosser L (2017) Assessment of endotracheal tube intubation. Review of existing scales. Disaster Emerg Med J 2(2):91-93. https://doi.org/10.5603/DEMJ.2017.0017

6. He W, Huang MM, Liu TS et al (2014) Comparison of HC videolaryngoscope and Macintosh direct laryngoscope for tracheal intubation in pediatric patients. Chin J Anesthesiol 34:1357-1360

7. Jagannathan N, Hajduk J, Sohn L, Huang A, Sawardekar A, Albers B, Bienia S, De Oliveira GS (2017) Randomized equivalence trial of the King Vision aBlade videolaryngoscope with the Miller direct laryngoscope for routine tracheal intubation in children $<2 \mathrm{yr}$ of age. Br J Anaesth 118(6):932-937. https://doi.org/10.1093/bja/ aex073

8. Jiang Y, Jin QY (2014) Comparison between home-made UE videolaryngoscope and direct laryngoscope for tracheal intubation in pediatric patients. J Chin Pract Diagn Ther 28:59-60

9. Karczewska K, Szarpak L, Smereka J, Dabrowski M, Ladny JR, Wieczorek W, Robak O, Frass M, Ahuja S, Ruetzler K (2017) ETView compared to direct laryngoscopy in patients with immobilized cervical spine by unexperienced physicians: a randomized crossover manikin trial. Anaesthesiol Intensive Ther 49(4):274-282. https://doi.org/10.5603/AIT.a2017.0047

10. Ladny JR, Sierzantowicz R, Kedziora J, Szarpak L (2017) Comparison of direct and optical laryngoscopy during simulated cardiopulmonary resuscitation. Am J Emerg Med 35(3):518-519. https://doi.org/10.1016/j.ajem.2016.12.026

11. Ladny JR, Smereka J, Szarpak L (2017) Comparison of the Trachway video intubating stylet and Macintosh laryngoscope for endotracheal intubation. Preliminary data. Am J Emerg Med 35(4): 574-575. https://doi.org/10.1016/j.ajem.2016.12.015

12. Madziala M (2018) The ETView tracheoscopic ventilation tube for trauma patient intubation. Disaster Emerg Med J 3(2):69-70. https://doi.org/10.5603/DEMJ.2018.0016

13. O'Shea JE, Thio M, Kamlin CO, McGrory L, Wong C, John J et al (2015) Videolaryngoscopy to teach neonatal intubation: a randomized trial. Pediatrics. 136:912-919

14. Parker MM, Nuthall G, Brown C 3rd, Biagas K, Napolitano N, Polikoff LA, Simon D, Miksa M, Gradidge E, Lee JH, Krishna AS, Tellez D, Bird GL, Rehder KJ, Turner DA, Adu-Darko M, Nett ST, Derbyshire AT, Meyer K, Giuliano J Jr, Owen EB, Sullivan JE, Tarquinio K, Kamat P, Sanders RC Jr, Pinto M, Bysani GK, Emeriaud G, Nagai Y, MA MC, Walson KH, Vanderford P, Lee A, Bain J, Skippen P, Breuer R, Tallent S, Nadkarni V, Nishisaki A, Pediatric Acute Lung Injury and Sepsis Investigators (PALISI) Network (2017) Relationship between adverse tracheal intubation associated events and PICU outcomes. Pediatr Crit Care Med 18(4):310-318. https://doi.org/10.1097/ PCC.0000000000001074

15. Pouppirt NR, Nassar R, Napolitano N, Nawab U, Nishisaki A, Nadkarni V, Ades A, Foglia EE (2018) Association between video laryngoscopy and adverse tracheal intubation-associated events in the neonatal intensive care unit. J Pediatr 201:281-284.e1. https:// doi.org/10.1016/j.jpeds.2018.05.046

16. Rabiner JE, Auerbach M, Avner JR, Daswani D, Khine H (2013) Comparison of GlideScope videolaryngoscopy to direct laryngoscopy for intubation of a pediatric simulator by novice physicians. Emerg Med Int 2013:407547-407546. https://doi.org/10.1155/ 2013/407547

17. Schulz KF, Altman DG, Moher D, CONSORT Group (2010) CONSORT 2010 statement: up-dated guidelines for reporting parallel group randomised trials. BMJ 340:c332. https://doi.org/10. 1136/bmj.c332

18. Shiima Y, Hsieh TC, Long A, Donoghue A (2018) Videographic assessment of pediatric tracheal intubation technique during emergency airway management. Pediatr Crit Care Med 19(3):e136e144. https://doi.org/10.1097/PCC.0000000000001423

19. Sinha PK, Mitra S, Gombar S, Gombar KK (1999) A new class for modified Mallampati sign. Anesth Analg 89(1):259-260

20. Smereka J, Czyzewski L, Szarpak L, Ladny JR (2017) Comparison between the TrueView EVO2 PCD and direct laryngoscopy for endotracheal intubation performed by paramedics: preliminary data. Am J Emerg Med 35(5):789-790. https://doi.org/10.1016/j. ajem.2016.11.063

21. Smereka J, Ladny JR, Naylor A, Ruetzler K, Szarpak L (2017) CMAC compared with direct laryngoscopy for intubation in patients with cervical spine immobilization: a manikin trial. Am J Emerg Med 35(8):1142-1146. https://doi.org/10.1016/j.ajem.2017.03.030

22. Sun Y, Lu Y, Huang Y, Jiang H (2014) Pediatric video laryngoscope versus direct laryngoscope: a meta-analysis of randomized controlled trials. Paediatr Anaesth 24(10):1056-1065. https://doi.org/ 10.1111/pan. 12458

23. Sunder RA, Haile DT, Farrell PT, Sharma A (2012) Pediatric airway management: current practices and future directions. Paediatr Anaesth 22(10):1008-1015. https://doi.org/10.1111/pan.12013

24. Szarpak L, Czyzewski L, Kurowski A, Truszewski Z (2015) Comparison of the TruView PCD video laryngoscope and Macintosh laryngoscope for pediatric tracheal intubation by novice paramedics: a randomized crossover simulation trial. Eur J Pediatr 174(10):1325-1332. https://doi.org/10.1007/s00431-015-2538-0

25. Szarpak L, Czyżewski L, Kurowski A (2015) Can GlideScope ${ }^{\circledR}$ videolaryngoscope be an alternative to direct laryngoscopy for child and infant tracheal intubation during chest compression? Eur J Pediatr 174(7):981-982. https://doi.org/10.1007/s00431-0152495-7 
26. Wan XX (2015) Comparative analysis of application of domestic UE videolaryngoscope and direct laryngoscope in pediatric tracheal intubation. Mod J Interg Tradit Chin Wes Med 24:1232-1234

27. Wang JL, Huang HQ, Shi XH, Fei J (2014) Clinical application of $\mathrm{HC}$ videolaryngoscope for intubation in the neonate with a cleft lip. J Clin Anesthesiol 30:785-787

28. Wojewodzka-Zelezniakowicz M, Madziala A, Madziala M (2017) Comparison of the Miller and Macintosh laryngoscopes in simulated pediatric trauma patient: a pilot study. Disaster Emerg Med J 2(1):1-6. https://doi.org/10.5603/DEMJ.2017.0001

29. Xue FS, Yang BQ, Liu YY, Li HX, Yang GZ (2017) Current evidences for the use of UEscope in airway management. Chin Med J 130(15):1867-1875. https://doi.org/10.4103/0366-6999.211536

30. Xue FS, Liu YY, Li HX, Yang GZ. Paediatric video laryngoscopy and airway management: what's the clinical evidence? Anaesth Crit
Care Pain Med. Available online 10 Jan 2018. https://doi.org/10. 1016/j.accpm.2017.11.018

31. Zheng Y (2014) Application of videolaryngoscope in tracheal intubation in neonates. Henan J Surg 20:30-32

32. Zheng P, He QW (2015) Application of HC videolaryngoscope in endotracheal intubation in children undergoing general anesthesia. Chin J Endosc 21:948-951

Publisher's note Springer Nature remains neutral with regard to jurisdictional claims in published maps and institutional affiliations.

\section{Affiliations}

\section{Jacek Smereka ${ }^{1} \cdot$ Marcin Madziala $^{2} \cdot$ Dominika Dunder $^{2} \cdot$ Elzbieta Makomaska-Szaroszyk $^{2} \cdot$ Lukasz Szarpak $^{2}$ iD}

1 Department of Emergency Medical Service, Wroclaw Medical University, Wroclaw, Poland
2 Lazarski University, 43 Swieradowska Str., 02-662 Warsaw, Poland 